\title{
Upregulation of a spliced variant of human interferon regulatory factor 3 through binding of the transcription factor Sp1 to the promoter
}

\author{
LIANG-HUA ZHU*, LING-ZHI QIU*, WEI REN and GUO-PING ZHOU \\ Department of Pediatrics, The First Affiliated Hospital of Nanjing Medical University, Nanjing, Jiangsu 210029, P.R. China
}

Received September 27, 2013; Accepted October 31, 2013

DOI: $10.3892 /$ br.2013.198

\begin{abstract}
Interferon regulatory factor 3 (IRF-3) plays an important role in host defense against viral and bacterial infection. IRF-3 includes a variety of spliced variants, which may regulate the transcription of IRF-3. We previously identified two novel IRF-3 spliced variants, Int2V1 and Int2V2, starting from intron 2 of the wild-type of IRF-3. However, the mechanism through which the IRF-3 spliced variants regulate transcription has not been elucidated. In this study, we demonstrated that the transcription factor Sp1 upregulates the basal transcriptional activity of IRF-3 Int2V1. By transient transfection analysis, we demonstrated that the overexpression of $\mathrm{Sp} 1$ led to positive regulation, whereas knocking down of the endogenous $\mathrm{Sp} 1$ resulted in repression of IRF-3 promoter activity. Electrophoretic gel mobility shift assays and chromatin immunoprecipitation assays demonstrated that Sp1 interacted with the IRF-3 promoter in vitro and in vivo. These results suggested that $\mathrm{Sp} 1$ positively regulated the transcription of a spliced variant of IRF-3 through directly binding to the $\mathrm{Sp} 1$ consensus binding site.
\end{abstract}

\section{Introduction}

Interferon (IFN) regulatory factors are a family of transcription factors that are crucial in the regulation of IFN-stimulated genes (ISGs) and the induction of type I IFNs, including IFN- $\alpha$ and IFN- $\beta$. The human interferon regulatory factor (IRF) family, which consists of 9 members (IRF1-9) (1), is defined by a highly conserved amino-terminal DNA-binding domain

Correspondence to: Dr Guo-Ping Zhou, Department of Pediatrics, The First Affiliated Hospital of Nanjing Medical University, 300 Guangzhou Road, Nanjing, Jiangsu 210029, P.R. China

E-mail: gpzhou2003@yeah.net

${ }^{*}$ Contributed equally

Key words: interferon regulatory factor 3, spliced variant, transcription, Sp1, reporter gene assay characterized by a 5-tryptophan residue repeat that allows the binding of IRFs, as homodimers or heterodimers, to consensus GAA and AANNNGAA motifs found in IFN-stimulated response elements (ISREs), including promoters of type I IFNs and ISGs (2). Each IRF also contains a unique C-terminal domain, referred to as the IRF association domain (IAD). This region was shown to be important in mediating IRF protein-protein interaction. The different IADs promote the interaction of IRFs with distinct transcription factors and contribute to the regulation of target genes $(3,4)$.

Alternative splicing is a general mechanism for regulating gene expression that affects the RNA products of $>90 \%$ of human genes (5). It is associated with numerous diseases, such as Kawasaki disease (6), systemic lupus erythematosus (7) and myasthenia gravis (8). IRF-3 includes a variety of spliced variants which may regulate the wild-type of IRF-3. IRF-3 isoforms produced by alternative splicing have been reported in humans (IRF-3a) and mice (mIRF-3a). The expression of IRF-3a results in potent and specific negative regulation of IRF-3 transcriptional activity (9). Additionally, Li et al (10) reported that the spliced variants IRF-3b, $-3 c,-3 d,-3 e$ and $-3 f$ were expressed in the majority of human cells and tissues and their expression was more frequent in tumor tissues compared to that in normal counterparts. It was reported that the ectopic expression of these spliced variants may inhibit the transactivation capacity of IRF-3 to varying degrees.

A previous study identified two novel spliced variants of IRF-3, starting from intron 2 of the wild-type of IRF-3, Int2V1 and Int2V2 (11). Their translation initiation ATG codons were found to be located 718 and $162 \mathrm{bp}$, respectively, upstream of the third exon. We reported that the transcription factor Sp1 upregulates the spliced variant Int2V1 (12), although the underlying molecular mechanism has not been fully elucidated. In this study, we demonstrated that exogenous Sp1 expression led to a significant increase in IRF-3 spliced variant mRNA expression and promoter activity. We investigated the molecular mechanism through which Sp1 upregulated the transcription of human IRF-3 gene through RNAi, electrophoretic gel mobility shift assays and chromatin immunoprecipitation assays and demonstrated that Sp1 upregulated IRF-3 spliced variant transcription through directly binding to the $\mathrm{Sp} 1$ consensus binding sequences at positions -130 to -120 bp relative to the transcriptional initiation site in the spliced variant of human IRF-3. 


\section{Materials and methods}

Cell culture. Human embryonic kidney (HEK)-293T cells (purchased from the Shanghai Chinese Academy of Sciences Institute for Cell Biology preserved by the State Key Laboratory of Reproductive Medicine, the First Affiliated Hospital of Nanjing Medical University, Nanjing, China) were maintained in Dulbecco's modified Eagle's medium (Thermo Fisher Scientific, Rockford, IL, USA) containing 10\% heat-inactivated fetal bovine serum (Zhejiang Tianhang Biological Technology Co., Ltd, Hangzhou, China) supplemented with penicillin $(100 \mathrm{U} / \mathrm{ml})$ and streptomycin $(100 \mu \mathrm{g} / \mathrm{ml})$. The cells were incubated at $37^{\circ} \mathrm{C}$ with $100 \%$ humidity in $5 \% \mathrm{CO}_{2}$ and passaged using standard cell culture techniques.

Plasmids, transfection and RNAi. The cloning of the spliced variant of human IRF-3 gene promoter region was performed as previously described(12). The expression plasmidspN3,pN3-Sp1, (donated by Dr Guntram Suske) (13) were cotransfected by using Lipofectamine $^{\mathrm{TM}} 2000$ (Invitrogen, Carlsbad, CA, USA) and then incubated for $24 \mathrm{~h}$. For the RNAi assay, the HEK-293T cells were transfected with Sp1 siRNA or control vector (primer of Sp1 siRNA: F, 5'-AUCACUCCAUGGAUGAAAUGATT-3' and R, 5'-UCSUUUCSUCCSUGGSGUGAUTT-3'; and primer of control: F, 5'-UUCUCCGAACGUGUCACGUTT-3' and R, 5'-ACGUGACACGUUCGGAGAATT-3') and cultured for $48 \mathrm{~h}$. The cells were then harvested to assess the effectiveness of RNA interference.

Dual-luciferase reporter assays. The HEK-293T cells were seeded in 96-well plates $24 \mathrm{~h}$ prior to transfection. Sp1 expression plasmid or empty vector was individually cotransfected into the cells, together with the appropriate IRF-3 spliced variant promoter reporter plasmids, using Lipofectamine 2000 (Invitrogen). For the RNAi assay, Sp1 siRNA or control vector was individually cotransfected into HEK-293T cells, with the appropriate IRF-3 spliced variant promoter reporter plasmids. The pRL-TK plasmid (2 ng/sample; Promega, Madison, WI, USA) containing the Renilla luciferase gene driven by the herpes simplex virus thymidine kinase promoter was cotransfected with the constructs and the luciferase activity was normalized. The preparation of cell lysates and measurements of luciferase activity were performed using the Dual Reporter Assay system (Promega) and the TD-20/20 luminometer (Turner Designs, Inc., Sunnyvale, CA, USA) according to the manufacturer's instructions.

Nuclear extract preparation and electromobility shift assay. Nuclear extracts from HEK-293T cells were prepared. Oligonucleotides (probes) were labeled at the 5' end with ATP using T4 polynucleotide kinase (Fermentas, Vilnius, Lithuania) . The proteins Sp1 (Promega) or nuclear extracts from the HEK-293T cells (100-300 ng) were pre-incubated in binding buffer (5X binding buffer, Promega) for $20 \mathrm{~min}$ at $25^{\circ} \mathrm{C}$ in a volume of $19 \mu \mathrm{l}$, with and without an excess of unlabeled oligonucleotide competitors. Following the addition of $1 \mu \mathrm{l}$ of labeled DNA, the mixture was incubated for 60-90 $\mathrm{min}$ at $4^{\circ} \mathrm{C}$. Each reaction mixture was then loaded into the well of a $4 \%$ non-denaturing polyacrylamide gel (Beyotime Institute of Biotechnology, Shanghai, China) and electrophoresed at
$100 \mathrm{~V}$ in $0.5 \mathrm{X}$ TBE buffer at $4^{\circ} \mathrm{C}$ for $1-2 \mathrm{~h}$. For competition experiments and antibody supershift, the competing unlabeled probes and antibodies were pre-incubated for $20 \mathrm{~min}$ at room temperature with the nuclear extracts prior to the addition of the radiolabeled probe. The DNA-protein complexes were resolved on $4 \%$ non-denaturing polyacrylamide gels for $3 \mathrm{~h}$ at $100 \mathrm{~V}$ in 0.5X TBE buffer. After electrophoresis, the gel was dried and exposed to autoradiography film at $-80^{\circ} \mathrm{C}$.

Chromatin immunoprecipitation assay (ChIP). The chromatin immunoprecipitation assay was performed using the ChIP-IT kit (Active Motif, Carlsbad, CA, USA) following the manufacturer's instructions. Briefly, three $100-\mathrm{cm}^{2}$ dishes of $80-90 \%$ confluent HEK-293T cells were treated with $1 \%$ formaldehyde in phosphate-buffered saline (PBS) for $10 \mathrm{~min}$ at room temperature. The formaldehyde was inactivated by the addition of $0.125 \mathrm{M}$ glycine in PBS to the cells for $5 \mathrm{~min}$ at room temperature. The cells were then washed in ice-cold PBS and lysed with lysis buffer containing $1 \%$ sodium dodecyl sulfate. Sonication of cross-linked chromatin was performed at $200 \mathrm{~W}$ with five rounds of 20 -sec pulses, so that the chromatin fragments thus obtained ranged from 500 to $1,000 \mathrm{bp}$ in size. Soluble chromatin was subjected to overnight immunoprecipitation with anti-IgG or anti-Spl (Santa Cruz Biotechnology, Santa Cruz, CA, USA). A portion of the chromatin solution was kept to measure the amount of input DNA in different samples prior to immunoprecipitation. For each immunoprecipitation, $2 \mu \mathrm{g}$ of the appropriate antibody was incubated with a precleared chromatin aliquot overnight at $4^{\circ} \mathrm{C}$. Following immunoprecipitation and elution, the eluent was heated to $65^{\circ} \mathrm{C}$ for $6 \mathrm{~h}$ to reverse the cross-link and DNA then was purified using the minicolumns provided with the kit. The purified DNA was amplified by the promoter-specific primers (ChIP-F, 5'-CACCCCTCGTCAACACCC-3' and ChIP-R, 5'-CGCGGGAAAGTTGAACTAATA-3'] and polymerase chain reaction (PCR) was performed under the following conditions: 1 cycle at $94^{\circ} \mathrm{C}$ for $5 \mathrm{~min} ; 36$ cycles of $30 \mathrm{sec}$ at $94^{\circ} \mathrm{C}$, $30 \mathrm{sec}$ at $59^{\circ} \mathrm{C}$ and $30 \mathrm{sec}$ at $72^{\circ} \mathrm{C}$; and a final extension step for $10 \mathrm{~min}$ at $72^{\circ} \mathrm{C}$. The PCR products were analyzed by electrophoresis on a $2 \%$ agarose gel.

$R N A$ purification and quantitative reverse trancription (qRT-PCR). Total RNA extraction was performed using TRIzol reagent (Invitrogen) followed by chloroform-isopropanol extraction and ethanol precipitation. Subsequently, duplicate samples of $1 \mu 1$ of each cDNA were used as a template. The quantification of gene transcripts was performed by qPCR using SYBR-Green I dye (Invitrogen) and the ABI PRISM 7700 Sequence Detection system (PE Applied Biosystems, Wellesley, MA, USA). The specificity of amplification was assessed for each sample by melting curve analysis. The expression values were normalized with control GAPDH. The primers used were as follows: spliced variant of IRF-3: sense primer, 5'-ACGGGATTAGACACCAAGTT-3' and antisense primer, 5'-TGGGATTACAGGCATGAGCT-3'; and GAPDH: sense primer, 5'-AGGTCGGAGTCAACGGAT-3' and antisense primer, 5'-TCCTGGAAGATGGTGATG-3'.

Statistical analysis. The results were analyzed by the paired two-tailed Student's-t test. $\mathrm{P}<0.05$ was considered to indicate a statistically significant difference. 
A

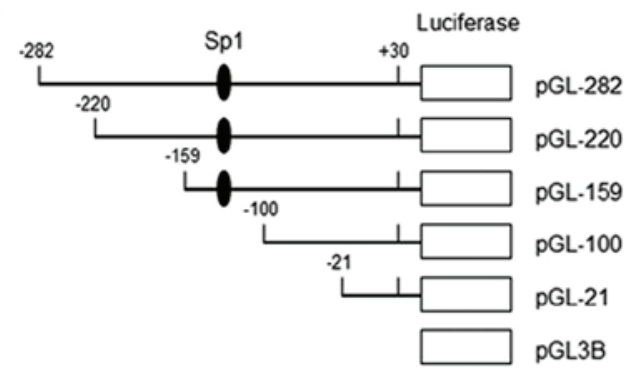

B

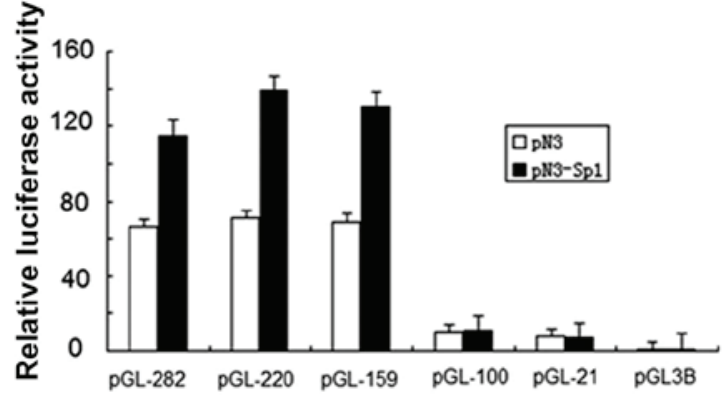

Figure 1. Effect of $\mathrm{Sp} 1$ overexpression on the interferon regulatory factor 3 (IRF-3) spliced variant promoter activity. (A) Schematic representation of various IRF-3 spliced variant promoter deletion constructs. The putative Sp1-binding site is indicated. (B) Sp1 expression plasmid and empty vector were transfected into HEK-293T cells together with each IRF-3 spliced variant promoter reporter plasmid. Equal concentrations of the Renilla reporter plasmid pRL-TK were also cotransfected as an internal control. The luciferase activity was measured and the relative fold activation of each IRF-3 spliced variant promoter normalized to the pRL-TK internal standard was presented in the histogram with arbitrary units. All the IRF-3 spliced variant promoter fragments, except pGL3-100 and pGL3-21, which do not have Sp1 binding sites, exhibited a significant upregulation under exogenous Sp1 expression.

\section{Results}

Overexpression of Sp1 leads to the upregulation of IRF-3 spliced variant promoter activity. To confirm the effect of Sp1 on IRF-3 spliced variant promoter activity, we investigated the effects of Sp1 overexpression on IRF-3 promoter activity and performed luciferase reporter assays with a series of IRF-3 spliced variant promoter deletion mutants (12). The schematic representation of the constructs used in this assay is presented in Fig. 1. Sp1 expression plasmid and empty vector were transfected into HEK-293T cells together with each IRF-3 promoter reporter plasmid indicated in Fig. 1B. Equal concentrations of Renilla reporter plasmid pRL-TK were also cotransfected as an internal control. Luciferase activity was measured and the relative fold activation of each IRF-3 spliced variant promoter normalized to the pRL-TK internal standard was presented in the histogram with arbitrary units. All the IRF-3 spliced variant promoter fragments, except pGL3-100 and pGL3-21, which do not have Sp1 binding sites, exhibited a significant upregulation under exogenous Sp1 expression (Fig. 1A). This result indicated that human IRF-3 spliced variant promoter was positively regulated by Sp1 in HEK-293T cells.

Knockdown of Spl expression by siRNA decreases IRF-3 spliced variant promoter activity. Our previous study demonstrated that a $\mathrm{Sp} 1$ binding site mutation resulted in a $50 \%$ decrease in the promoter activity compared to that of the unmodified promoter of Sp1 (12). To further confirm the

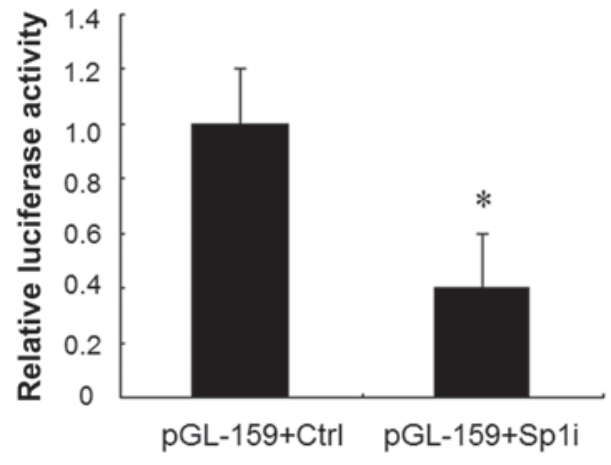

Figure 2. Knockdown of Sp1 expression by siRNA decreases interferon regulatory factor 3 (IRF-3) spliced variant promoter activity. The HEK-293T cells were cotransfected with the reporter plasmids pGL-159 and Sp1 siRNA or control vector. The relative luciferase activity of the IRF-3 spliced variant promoter controlled by reporter plasmid pGL-159 cotransfected with negative control was set as 1 ( ${ }^{*} \mathrm{P}<0.05$ vs. control). All the experiments were performed in triplicate. The data from the experiments and their average are indicated in the figure. Error bars, standard deviation. Ctrl, control.

role of Sp1 in the regulation of IRF-3 spliced variant promoter activity, we used RNA interference to knock down Sp1 expression in HEK-293T cells. We cotransfected the reporter plasmids pGL3-159 together with Sp1 siRNA or control vector into HEK-293T cells individually. A 60\% decrease of luciferase activity was observed in the presence of Sp1 siRNA compared to that observed with the control vector (Fig. 2). These results further validated that Sp1 upregulated the transcriptional activity of the IRF-3 spliced variant promoter.

Spl binds to the IRF-3 spliced variant promoter in vitro and in vivo. An electrophoretic mobility shift assay was performed to investigate the possibility of Sp1 directly binding to and upregulating the IRF-3 spliced variant promoter. A wild-type oligonucleotide containing the Sp1 consensus binding site (GGGGGATGGT) in the context of the IRF-3 promoter was synthesized. The oligonucleotide was biotin-labeled and incubated with the nuclear protein from HEK-293T cells. The nuclear protein bound to the wild-type oligonucleotide, forming a protein-DNA complex (Fig. 3A, lane 2). By contrast, there was no protein-DNA complex formation when no nuclear extract was incubated with the wild-type oligonucleotides (Fig. 3A, lane 1). Competition assays (using 10- and 100-fold excess of cold competitor oligonucleotides) confirmed the absence of protein-DNA complex formation (Fig. 3A, lanes 3 and 4). The composition of this protein-DNA complex was investigated by the addition of antibodies against Sp1. Thus, Sp1 may upregulate the IRF-3 spliced variant via directly binding to its promoter.

To determine whether Sp1 binds to the IRF-3 promoter in vivo, we performed a ChIP assay (Fig. 3B), which was used for the detection of proteins and the specific regions of DNA binding in vivo. The HEK-293T cells were fixed with formaldehyde, lysed and the chromatin was cleaved by nuclease digestion. The chromatin was then immunoprecipitated with anti-IgG and anti-Spl antibodies and the DNA precipitated in the complexes was subjected to PCR amplification with primers flanking the region containing the Spl-binding site. The results indicated that Sp1 may directly interact with the IRF-3 promoter in vivo. 
A

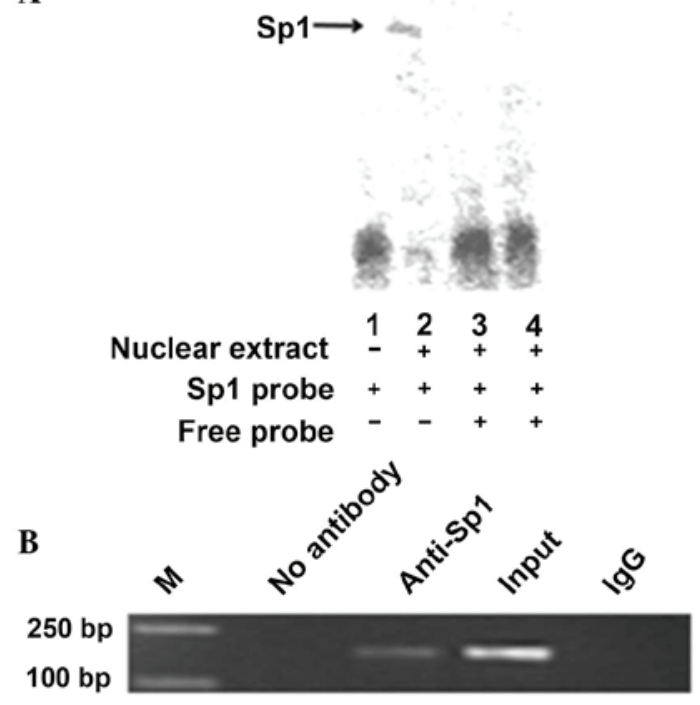

Figure 3. Sp1 binds to the interferon regulatory factor 3 (IRF-3) spliced variant promoter in vitro and in vivo. (A) An electrophoretic mobility shift assay was performed to assess whether $\mathrm{Sp} 1$ directly binds and thereby upregulates the IRF-3 spliced variant promoter. The nuclear protein bound to the wild-type oligonucleotide, forming a protein-DNA complex (lane 2). By contrast, there was no protein-DNA complex formation when no nuclear extract was incubated with the wild-type oligonucleotides (lane 1). (B) A chromatin immunoprepitation (ChIP) assay was performed to confirm the interaction of Sp1 with the IRF-3 spliced variant promoter in vivo. ChIP analysis was performed in Sp1 overexpressing HEK-293T cells. Input: the sonicated cell lysate was diluted 1:10 and used for PCR. Preciptation with an unrelated antibody ( $\mathrm{IgG})$ was used as negative control. The PCR products from the ChIP assay were run on an agarose gel.

A
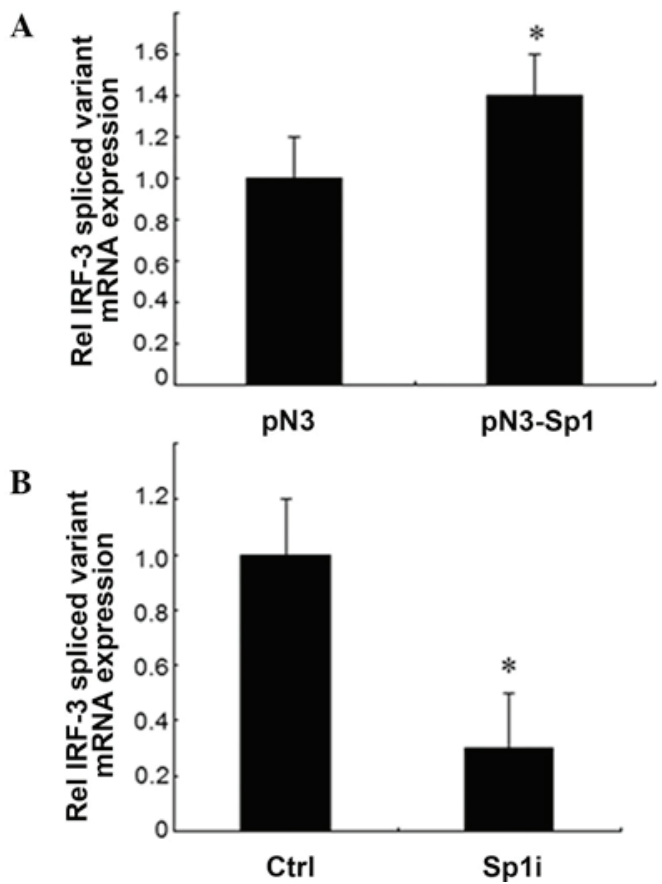

Figure 4. Quantitative RT-PCR analysis of the interferon regulatory factor 3 (IRF-3) spliced variant mRNA content of HEK-293T cells. (A) Quantification of IRF-3 spliced variant at the mRNA level following overexpression of Sp1 ( ${ }^{*}<0.05$ vs. pN3). (B) Quantification of IRF-3 at the mRNA level following siRNA-mediated knockdown of $\mathrm{Sp} 1$ (" $\mathrm{P}<0.05$ vs. control). All the experiments were performed in triplicate. The data from the experiments and their average are indicated in the figure. Error bars, standard deviation. Ctrl, control.
Spl leads to the increase of the expression of IRF-3 spliced variant $m R N A$. To further investigate whether Sp1 affected the expression of IRF-3, the mRNA levels of the IRF-3 spliced variant were determined in HEK-293T cells following transient transfection with the $\mathrm{Sp1}$ expression vector or siRNA against $\mathrm{Sp} 1$ by qRT-PCR. The transient expression of Sp1 resulted in a $40 \%$ increase of IRF-3 spliced variant mRNA expression compared to the control vector (Fig. 4A). By contrast, the use of Sp1 siRNA resulted in a 70\% reduction of IRF-3 mRNA expression compared to the control vector (Fig. 4B). These data demonstrated that $\mathrm{Sp1}$ upregulated the expression of endogenous IRF-3 gene at the level of transcription.

\section{Discussion}

Alternative splicing is a key mechanism for expanding transcript and protein diversity of mammalian genes. Several diseases are associated with alternative splicing. Kappova et al (9) described a second mRNA that was generated from the IRF-3 gene by alternative splicing and demonstrated that alternative splicing of the IRF-3 gene-encoded transcript resulted in the production of two isoforms with antagonistic functions. It was also reported that the relative value of IRF-3 and IRF-3a exerted an effect on carcinogenesis (14). We previously identified two new spliced variants of IRF-3, referred to as Int2V1 and Int2V2. By generating a series of 5' deletions, we demonstrated that the Int $2 \mathrm{~V} 1$ core promoter located within the region -159/-100 bp relative to the TSS and Sp1 transcription factor positively regulated the human IRF-3 gene promoter. In this study, we aimed to investigate the molecular mechanism underlying the upregulation of promoter activity by Sp1.

Transcription factors may regulate target genes by direct or indirect interaction with their promoters. The transcription factor E2F1 represses the expression of IRF-3 by directly binding to its promoter $(15,16)$. The CCAAT/enhancer-binding protein (C/EBP) family of transcription factors augments proximal and distal promoter activation of LMP1 by binding to a motif in the proximal promoter (17). The Epstein-Barr virus immediate-early replication and transcription activator (Rta) protein was shown to regulate the BRLF1 gene by indirect interaction through the formation of an Sp1-MCAF1-Rta complex at Sp1 sites (18).

$\mathrm{Sp} 1$ is a ubiquitous nuclear factor that plays a key role in maintaining basal transcription of house-keeping genes. It was demonstrated that $\mathrm{Sp} 1$ is involved in numerous cellular processes, such as cell growth and differentiation. Sp1 is also crucial in the growth and metastasis of several tumors by regulating oncogenes, tumor suppressor genes, cell cycle control molecules, growth-related signal transduction, angiogenesis-related factors and apoptosis (19).

The Sp1 transcription factor regulates gene expression through multiple mechanisms. Sp1 binds to GC-rich motifs with high affinity and may regulate the expression of TATA-containing and TATA-deficient genes via protein-protein interactions or interaction with other transcription factors. It was reported that Sp1 may be asociated with chromatin remodeling through interactions with chromatin-modifying factors, such as p300 (20) and histone deacetylases (21). We recently demonstrated that the transcriptional factors $\mathrm{Sp} 1$ and $\mathrm{Sp} 3$ bound to the $\mathrm{CD} 2 \mathrm{AP}$ minimal promoter region and increased $\mathrm{CD} 2 \mathrm{AP}$ 
expression at the mRNA level in HEK-293 cells (22). In this study, we demonstrated that the $\mathrm{Sp} 1$ protein bound to the Sp1 consensus binding site in the IRF-3 promoter in vitro by electrophoretic gel mobility shift assays and antibody competition assays. Chromatin immunoprecipitation assays also demonstrated that $\mathrm{Sp} 1$ interacted with the IRF-3 promoter in vivo.

In summary, our studies identified a cis-regulatory element within the spliced variant of the human IRF-3 gene promoter and demonstrated that Sp1 directly bound to this cis-regulatory element and the exogenous expression of Sp1 significantly upregulated the transcription of the IRF-3 spliced variant. Characterizing the molecular regulation of human IRF-3 and the transcription of its spliced variants may be an important step towards elucidating the key role of IRF-3 in host defense against viral and bacterial infection and cell growth regulation.

\section{Acknowledgements}

This study was supported by grants from the National Natural Science Foundation of China (nos. 30570863 and 30872804), the Natural Science Foundation of Jiangsu Province, China (no. BK2007244) and the Medical Academic Key Talent Program of Jiangsu Province, China (no. RC2007050).

\section{References}

1. Taniguchi T, Ogasawara K, Takaoka A and Tanaka N: IRF family of transcription factors as regulators of host defense. Annu Rev Immunol 19: 623-655, 2001.

2. Paun A and Pitha PM: The IRF family, revisited. Biochimie 89: 744-753, 2007.

3. Mamane Y, Heylbroeck C, Genin P, et al: Interferon regulatory factors: the next generation. Gene 237: 1-14, 1999.

4. Takaoka A, Tamura $\mathrm{T}$ and Taniguchi $\mathrm{T}$ : Interferon regulatory factor family of transcription factors and regulation of oncogenesis. Cancer Sci 99: 467-478, 2008.

5. Evsyukova I, Somarelli JA, Gregory SG and Garcia-Blanco MA Alternative splicing in multiple sclerosis and other autoimmune diseases. RNA Biol 7: 462-473, 2010.

6. Onouchi Y, Gunji T, Burns JC, et al: ITPKC functional polymorphism associated with Kawasaki disease susceptibility and formation of coronary artery aneurysms. Nat Genet 40: 35-42, 2008.

7. Kozyrev SV, Abelson AK, Wojcik J, et al: Functional variants in the B-cell gene BANK1 are associated with systemic lupus erythematosus. Nat Genet 40: 211-216, 2008.
8. Brenner T, Hamra-Amitay J, Evron T, Boneva N, Seidman S and Soreq H: The role of readthrough acetylcholinesterase in the pathophysiology of myasthenia gravis. FASEB J 17: 214-222, 2003.

9. Karpova AY, Ronco LV and Howley PM: Functional characterization of interferon regulatory factor $3 \mathrm{a}$ (IRF-3a), an alternative splice isoform of IRF-3. Mol Cell Biol 21: 4169-4176, 2001.

10. Li Y, Hu X, Song Y, Lu Z, Ning T, Cai H and Ke Y: Identification of novel alternative splicing variants of interferon regulatory factor 3. Biochim Biophys Acta 1809: 166-175, 2011.

11. Ren W, Xu HG, Lu C, Jin R, Zou L, Wang Y and Zhou GP: The characterization of two novel IRF-3 transcripts starting from intron 2 of the wild type of IRF-3. Mol Biol Rep 38: 4415-4421, 2011.

12. Ren W, Zhu LH, Xu HG, Jin R and Zhou GP: Characterization of a spliced variant of human IRF-3 promoter and its regulation by the transcription factor Sp1. Mol Biol Rep 39: 6987-6993, 2012.

13. Krüger I, Vollmer M, Simmons DG, Elsässer HP, Philipsen S and Suske G: Sp1/Sp3 compound heterozygous mice are not viable: impaired erythropoiesis and severe placental defects. Dev Dyn 236: 2235-2244, 2007.

14. Bourdon JC, Fernandes K, Murray-Zmijewski F, et al: p53 isoforms can regulate p53 transcriptional activity. Genes Dev 19: 2122-2137, 2005

15. Xu HG, Ren W, Lu C and Zhou GP: Characterization of the human IRF-3 promoter and its regulation by the transcription factor E2F1. Mol Biol Rep 37: 3073-3080, 2010.

16. Xu HG, Ren W, Zou L, Wang Y, Jin R and Zhou GP: Direct repression of the human IRF-3 promoter by E2F1. Immunogenetics 63: 189-196, 2011.

17. Noda C, Murata T, Kanda T, et al: Identification and characterization of CCAAT enhancer-binding protein (C/EBP) as a transcriptional activator for Epstein-Barr virus oncogene latent membrane protein 1. J Biol Chem 286: 42524-42533, 2011.

18. Chang LK, Chung JY, Hong YR, Ichimura T, Nakao M and Liu ST: Activation of Sp1-mediated transcription by Rta of Epstein-Barr virus via an interaction with MCAF1. Nucleic Acids Res 33: 6528-6539, 2005.

19. Sankpal UT, Goodison S, Abdelrahim M and Basha R: Targeting Sp1 transcription factors in prostate cancer therapy. Med Chem 7: 518-525, 2011.

20. Suzuki T, Kimura A, Nagai R and Horikoshi M: Regulation of interaction of the acetyltransferase region of p300 and the DNA-binding domain of Sp1 on and through DNA binding. Genes Cells 5: 29-41, 2000.

21. Zhao S, Venkatasubbarao K, Li S and Freeman JW: Requirement of a specific Spl site for histone deacetylase-mediated repression of transforming growth factor beta type II receptor expression in human pancreatic cancer cells. Cancer Res 63: 2624-2630, 2003.

22. Xu HG, Ren W, Zou L, Wang Y, Jin R and Zhou GP: Transcriptional control of human CD2AP expression: the role of Sp1 and Sp3. Mol Biol Rep 39: 1479-1486, 2012. 